\title{
Sleep apnea and pregnancy. An association worthy of study
}

\author{
Felix del Campo • Carlos Zamarrón
}

Received: 3 July 2012 /Revised: 8 July 2012 / Accepted: 11 July 2012 / Published online: 9 August 2012

(C) Springer-Verlag 2012

Pregnancy involves major physiological and hormonal changes that have important implications in many areas of health, including sleep. Reports exist during pregnancy, predominantly in the first and last trimesters, describing changes in the quality and quantity of sleep [1]. Increased prevalence of snoring and excessive daytime sleepiness has also been described [2]. Sleepiness is a prevalent symptom in the general population, although it is difficult to assess objectively. Its presence is characteristic of obstructive sleep apnea syndrome (OSAS) but not exclusive to it. In fact, OSAS patients have a diverse phenotypic expression and do not always present this symptom. Excessive daytime sleepiness presents low sensitivity and specificity for the diagnosis of OSAS.

In the study published in this issue of Sleep and Breathing, Bourjeily et al. [3] point out that daytime sleepiness may not always be considered physiologically normal during pregnancy and may be due to other factors, including sleep-disordered breathing. The presence of other associated symptoms, such as snoring or apneic breathing, should raise suspicion.

The prevalence and severity of OSAS in pregnant women have not been clearly established. This is partly because the disease has historically received little attention in the field of obstetrics and because polysomnographic studies are difficult to accept during pregnancy. The majority of studies

F. del Campo $(\bowtie)$

Departmento de Medicina, Dermatología y Toxicología

de la Facultad de Medicina, Hospital Río Hortega,

Universidad de Valladolid,

Valladolid, Spain

e-mail: fsas@telefonica.net

\section{Zamarrón}

Division of Respiratory Medicine, Hospital Clínico Universitario,

Santiago de Compostela, Spain

e-mail: Carlos.Zamarron.Sanz@sergas.es during pregnancy focus on snoring [4]. However, in recent years, there has been an increased interest regarding the presence of OSAS in pregnant women [5-7] as a result of its potential impact in areas such as gestational hypertension, gestational diabetes, and fetal damage $[8,9]$.

The presence of OSAS in pregnant patients raises important issues. Firstly, there is some debate with respect to diagnostic procedure. Various alternative approaches have been proposed to facilitate diagnosis during consultations. The usual clinical questionnaires are less useful in these patients, so overnight polysomnography remains the gold standard for OSAS diagnosis. Simplified sleep studies such as pulse oximetry are not useful because respiratory events will have a greater impact on fragmentation (arousals) than on oxyhemoglobin saturation [9]. Questionnaires and sleep apnea detection devices have not been validated for pregnant women. Consequently, long-term research is necessary to be performed to find a reliable and effective screening tool in these patients.

Another point of contention is whether OSAS is a temporary phenomenon that will disappear after childbirth [10] or, on the other hand, if it is a chronic condition that will persist into the future. In this context, we should keep in mind the role of hormonal changes and weight gain during pregnancy, as well as the importance of obesity as a confounding variable. It seems reasonable that patients diagnosed with OSAS before pregnancy should be followed up for possible complications after childbirth. Moreover, these patients require monitoring in case of any surgery during childbirth [11].

Another aspect for consideration is the issue of CPAP treatment for pregnant OSAS patients. There is little research available regarding this topic, although CPAP appears to be effective and safe [12]. In particular, CPAP has been effective in pregnant patients with preeclampsia risk to help control blood pressure [7]. Another question is if 
the same criteria for treatment with CPAP should be used as in non-pregnant OSAS patients.

Lastly, another area of great interest in this population is the potential consequences of OSAS for the fetus [13]. OSAS has been associated with a deceleration of heart rate, lower Apgar score, an enhanced risk of preterm birth, and lower fetus weight. Further long-term prospective studies are necessary to assess the impact of this disease on the fetus.

In conclusion, sleep disorders such as snoring, excessive daytime sleepiness, and poor sleep are frequent during pregnancy. Although sometimes considered normal, it should be taken into account that certain symptoms may be associated to something beyond pregnancy and may involve associated complications that require a correct diagnosis and treatment.

Conflict of interest The authors declare that they have no conflict of interest.

\section{References}

1. Miller MA, Ahuja M, Cappuccio FP (2012) Sleep and pregnancy: sleep deprivation, sleep disturbed breathing and sleep disorders in pregnancy. Sleep Disorders, Chris Idzikowski (ed). ISBN: 978953-51-0293-9, InTech

2. Guilleminault C, Querra-Salva MA, Chowdhuri S, Poyares D (2000) Normal pregnancy, daytime sleeping, snoring and blood pressure. Sleep Med 1:289-297
3. Bourjeily G, Raquer CH, Chalhoub M, Miller M (2012) Excessive daytime sleepiness in late pregnancy may not always be normal: results from a cross sectional study. Sleep Breathing (manuscript number: SLBR-D-12-00007R1). doi:10.1007/s11325-012-0753-8

4. Tauman R, Many A, Deutsch V, Arvas S, Ascher-Landsberg J, Greenfeld M, Sivan Y (2011) Maternal snoring during pregnancy is associated with enhanced fetal erythropoiesis - a preliminary study. Sleep Med 12(5):518-522

5. Bourjeily G, Ankner G, Mohsenin V (2011) Sleep-disordered breathing in pregnancy. Clin Chest Med 32:175-189

6. Naud K, Ouellet A, Brown C, Pasquier JC, Moutquin JM (2010) Is sleep disturbed in pregnancy? J Obstet Gynaecol Can 32(1):28-34

7. Champagne KA, Kimoff RJ, Barriga PC, Schwartzman K (2010) Sleep disordered breathing in women of childbearing age \& during pregnancy. Indian J Med Res 131:285-301

8. Fung AM, Wilson DL, Barnes M, Walker SP (2012) Obstructive sleep apnea and pregnancy: the effect on perinatal outcomes. J Perinatol 32(6):399-406

9. Reid J, Skomro R, Cotton D, Ward H, Olatunbosun F, Gjevre J, Guilleminault C (2011) Pregnant women with gestational hypertension may have a high frecuency of sleep disordered breathing. Sleep 34(8):1033-1038

10. Edwards N, Blyton D, Hennessy A (2005) Severity of sleep disordered breathing improves following parturition. Sleep 28:737-741

11. Louis J, Auckley D, Bolden N (2012) Management of obstructive sleep apnea in pregnant women. Obstet Gynecol 119 (4):864-868

12. Guilleminault C, Kreutzer M, Chang JL (2004) Pregnancy, sleep disordered breathing and treatment with nasal continuous positive airway pressure. Sleep Med 5(1):43-51

13. Sahin FK, Koken G, Cosar E, Saylan F, Fidan F, Yilmazer M, Unlu M (2008) Obstructive sleep apnea in pregnancy and fetal outcome. Int J Gynaecol Obstet 100(2):141-146 